\title{
Evaluación de riesgos laborales en las actividades de maniobra convencional en el Perú
}

\author{
Evaluation of occupational risks in conventional maneuvering activities in \\ Peru
}

\author{
Michael Omar Padilla García ${ }^{1}$, Oskar Michael Huapaya Ramírez ${ }^{2}$
}

Recibido: 01/07/2020 - Aprobado: 30/10/2020 - Publicado: 30/11/2020

\begin{abstract}
RESUMEN
La presente investigación tuvo como objetivo evaluar los riesgos laborales a los que se exponen los trabajadores que realizan actividades de maniobra convencional. Maniobra convencional es el proceso de elevación y posicionamiento de equipos, componentes o materiales; ejecutado con las manos o con la ayuda de equipos o dispositivos mecánicos controlados de forma manual. Este estudio de tipo descriptivo se realizó en tres trabajos más representativos de maniobra convencional para la empresa Stierlift SA, una de las más representativas en el rubro. La metodología utilizada incluyó: la observación del trabajo, entrevista a los trabajadores y a los representantes de la empresa, la aplicación de encuestas para evaluar las condiciones de trabajo e infraestructura, la identificación de peligros, y finalmente, la evaluación de los riesgos laborales. Los resultados muestran que los trabajadores están expuestos principalmente un nivel de riesgo de medio hasta alto; mientras que, en promedio de los tres trabajos de maniobra considerados en el presente estudio, se obtiene un $32 \%$ de riesgos de nivel alto o significativo.
\end{abstract}

Palabras clave: Accidentes; evaluación; maniobra convencional; plan de acción; riesgos laborales; significativo.

\begin{abstract}
The present research aimed to evaluate the occupational risks to which workers who carry out conventional maneuvering activities are exposed. Conventional maneuvering is the process of lifting and positioning equipment, components, or materials; executed with the hands or with the aid of manually controlled mechanical equipment or devices. This descriptive study was carried out in three most representative works of conventional maneuvering for the company Stierlift SA, one of the most representative in the field. The methodology used included: job observation, interviewing workers and company representatives, applying surveys to evaluate working conditions and infrastructure, identifying hazards, and finally, evaluating occupational hazards. The results show that workers are mainly exposed to a medium to high level of risk; while, on average of the three maneuvering tasks considered in the present study, a $32 \%$ risk of high or significant level is obtained.
\end{abstract}

Keywords: Accidents; evaluation; conventional maneuver; action plan; occupational risks; significant.

\footnotetext{
1 Egresado de la Maestría en Gestión Integrada en Seguridad, Salud ocupacional y medio Ambiente, Facultad de Ingeniería Geológica, Minera, Metalúrgica y Geográfica, Universidad Nacional Mayor de San Marcos. Lima, Perú.

Autor para correspondencia: opadilla1089@gmail.com ORCID: https://orcid.org/0000-0002-5899-8868

2 Docente de la Facultad de Ingeniería Geológica, Minera, Metalúrgica y Geográfica, Universidad Nacional Mayor de San Marcos. Lima, Perú. E-mail: oskar.huapaya@gmail.com
} 


\section{INTRODUCCIÓN}

La presente investigación tiene como objetivo determinar el grado de influencia de la evaluación de riesgos laborales en la reducción de accidentes en las actividades de maniobra convencional en el Perú. La hipótesis que se busca comprobar es; si la evaluación de riesgos laborales influye significativamente en la gestión para la reducción de accidentes de maniobra convencional en el Perú.

En los últimos años a partir de la creación de la Sunafil y la ejecución de su acción orientadora y fiscalizadora, la seguridad y salud en el trabajo viene progresando positivamente; sin embargo, este desarrollo no ha sido suficiente, pues persiste una alta tasa de accidentes de trabajo (Del Águila Guerrero, 2020).

En muchos casos los empleadores de las PYMES al desconocer los beneficios de la implementación de un sistema de SST y el estudio de riesgos, como son la reducción de los accidentes y enfermedades, no toman la decisión de incluirlos en su productividad y rentabilidad (Falconi Agapito \& Romero Baylon, 2020).

Parte de lo que se evalúa en el sistema de gestión integrado es la presencia de riesgos y eso se observa en la gestión (Flores Arévalo, 2019).

El trabajo de maniobra convencional consiste en el proceso de elevación y posicionamiento de equipos, componentes o materiales; ejecutado con las manos o con la ayuda de equipos, como tacos de madera, gatas hidráulicas, uso de Ganttry, dispositivos de amarre y otros equipos (The Bechtel Equipment Operations Department Rigging, 2002). Este tipo de actividades representan un mayor esfuerzo físico, con movimientos repetitivos, riesgos de golpes y atrapamientos con la carga, por lo que requiere de una atención y evaluación de los riesgos (The Bechtel Equipment Operations Department Rigging, 2002). Sin embargo, en la actualidad y en el Perú no se tiene una norma que determine o especifique estándares mínimos, que se debieran cumplir para ejecutar las actividades de maniobra de forma segura, por esta razón, las empresas que desarrollan estos trabajos tienen que basarse en normas internacionales y adaptarlas a la realidad del país y del entorno. Al no existir normas técnicas de las actividades de maniobra, las empresas que brindan estos servicios tienen que guiarse de estándares sectoriales relacionados a estas tareas, por lo que se dificulta tomar controles específicos, para la disminución de los riesgos propios de la tarea de maniobra convencional (Stierlift SA., 2019).

Uno de los riesgos generados en este sector, está dado por la actividad física forzada por el levantamiento de carga y materiales de maniobra, ocasionando problemas de postura, lumbalgia y riesgo de golpes, choques o atropellos debido a que en su mayoría esta actividad se realiza en áreas transitadas (Consejería de Economía y empleo de España, 2008).

La actividad de maniobra convencional en el Perú, es uno de los trabajos con mayor demanda para el traslado y movimiento de cargas de grandes dimensiones y peso, lo cual hace de esta labor una tarea indispensable. Hay empresas especializadas en los trabajos de maniobra de carga, siendo la empresa Stierlift SA la más representativa por ser la pionera en realizar este servicio en el Perú (Stierlift SA., 2019).

Esta empresa actualmente desarrolla la actividad de maniobra convencional en distintos lugares del Perú, y tiene como clientes a compañías de los diversos sectores para el levantamiento y posicionamiento de grandes equipos y estructuras. Realizan trabajos de maniobra convencional que involucra una serie de riesgos a la seguridad y salud de los trabajadores, los cuales al ser repetitivos pueden ocasionar consecuencias como accidentes y enfermedades ocupacionales, siendo los principales riesgos que esta actividad genera los siguientes: caídas de personas a distinto nivel, choque y atropello, golpes, atrapamiento, tendinitis, sinovitis, lumbalgia, lesión de la conjuntiva, lesión dérmica, caída de la carga, tomar posturas incorrectas, realizar movimientos repetitivos y bruscos (Stierlift SA., 2019).

En la actualidad no se ha realizado estudios de los riesgos en las actividades de maniobra convencional en el Perú, se ha tomado referencia de estudios internacional para mitigar los riesgos de estas actividades, por lo que el índice de accidentabilidad es considerable.

\section{METODOS}

\subsection{Selección de muestra}

Fueron seleccionados todos los trabajadores que participan en los diversos trabajos de maniobra convencional como son: supervisor de maniobra, maniobristas, operadores y ayudantes; como muestra representativa los trabajadores seleccionados son los que se encuentran en planilla 2019 de la empresa Stierlift SA, siendo en total 20 personas.

\subsection{Técnicas de recolección de datos}

Se utilizó la técnica cuantitativa para el desarrollo del trabajo, ya que según Hernández Sampieri et al. (2014) en "la técnica cuantitativa se procede a la recopilación de datos, apoyado de resultados de análisis con base en medición numérica con el fin de probar las hipótesis establecidas, por medio de la observación, encuesta, entrevista" (p. 148).

Se tomó como análisis tres trabajos de maniobra convencional más representativos de la empresa Stierlift SA, especialista en estos servicios, en las cuales se combinan algunos de los diferentes tipos de maniobra y sus elementos los cuales son:

- Maniobra autodescarga y posicionamiento de transformador sobre pedestal.

- Maniobra posicionamiento de locomotora con Gantry

\section{- Maniobra descarga de vagones de tren Línea 2}

Se recopiló datos utilizando la observación, inspecciones y auditorias en el campo durante el desarrollo de las actividades de maniobra convencional y para esto se utilizarán instrumentos como información de las 
actividades de la empresa de muestra, recursos materiales como útiles y documentación base como manuales, guías o normas. Se realizó también encuestas y entrevistas a los trabajadores involucrados en dichas tareas, se revisarán datos estadísticos de accidentabilidad, casi accidentes, horas hombre, tiempo de trabajo, enfermedades ocupacionales, descansos médicos, ausencias laborales, opiniones del médico ocupacional, esta información será proporcionada por la empresa muestra Stierlift SA la cual brindará las facilidades tanto de documentación como visitas en sus instalaciones.

Se determinó la mejor metodología de evaluación, una matriz de riesgos basada en el DS N024-2016-EM (Diario El Peruano, 2016) debido a que los servicios que de maniobra de la empresa Stierlift son en su mayoría realizados en instalaciones mineras; se plasmará la información antes recabada identificando lo peligros y sus riesgos y se valorará teniendo en cuenta los antecedentes de accidentes e incidentes relacionados a los riesgos encontrados, historial de enfermedades ocupacionales o ausencia por salud, experiencia de los trabajadores obtenida de la entrevista, estándares de trabajo y legislación o normas vigentes.

Primero se efectuó un diagnóstico línea base de la actividad de maniobra convencional.

Se realizó una encuesta a todos los trabajadores de la muestra, un cuestionario en el cual contiene preguntas de su ambiente laboral, las condiciones, sus peligros y riesgos que considere.

Se llevó a cabo una observación de todas las actividades y el desarrollo en campo de cada variante acompañado del responsable y especialista de las actividades, se entrevistó a los trabajadores de los puestos involucrados.

Luego se tomó los antecedentes de enfermedades ocupacionales de los últimos 2 años y accidentes anteriores de los últimos 3 años.

Para obtener resultados de cuantos accidentes o enfermedades ocupacionales se ha tenido para el personal involucrado en una maniobra, se ha determinado por medio del método de recopilación de información en base a la solicitud de datos estadísticos y antecedentes a la empresa en estudio Stierlift SA y a la entrevista con el medico ocupacional de la empresa, esta información tendrá que ser de los 3 últimos años, para el caso de accidentes la información será según el DS024-2016.

Posteriormente se determinó una metodología para la evaluación de riesgos basada en el cumplimiento de la legislación y que sea adecuada para mostrar los resultados propuestos.

\subsection{Método de Análisis de riesgos a la seguridad}

Se utilizó el método de evaluación de riesgos según el Decreto Supremo No 024-2016-EM (Diario El Peruano (2016) ya que los trabajos mencionados que serán sujeto de análisis son realizados dentro de instalaciones mineras ya que los clientes que presenta la empresa de estudio Stierlift tienen en su mayoría clientes mineros. 2016 :

Método de evaluación de riesgos según el DS 024-

Es un método basado en la magnitud del riesgo y su jerarquización según la importancia de sus consecuencias (Severidad), responde a una relación matemática básica y sencilla donde el Nivel del Riesgo es igual al producto de los factores $\mathrm{P}, \mathrm{S}$.

$$
\mathrm{R}=\mathrm{P} \times \mathrm{S}
$$

Probabilidad (P): Posibilidad de que algo suceda, accidente o enfermedad, bajo ciertas condiciones técnicas y de proceso.

Severidad (S): Valoración de daños posibles debido a un accidente o enfermedad.

\section{Nivel de Riesgo (R): Valor del riesgo}

Para su confección se utilizan valores de referencia y estadísticas históricas, según el DS024-2016 en el anexo 7 se propone las tablas 1 y 2 para los criterios de Probabilidad, Severidad y nivel de riesgo.

Manejando cada tabla para cada riesgo y utilizando la formula anterior, se obtiene el valor de la magnitud del riesgo, que permite comparar diferentes riesgos.

El método elegido según el DS 024-2016 EM, permitió procesar información en primer tiempo sobre los agentes de riesgo, que podrán ocasionar una lesión, daño o enfermedad en las diferentes etapas del proceso.

Tabla 1. Criterios de severidad y probabilidad o frecuencia

\begin{tabular}{|c|c|c|c|c|c|c|c|}
\hline \multirow{5}{*}{$\begin{array}{l}\text { 是 } \\
\text { 䍃 } \\
\text { 怠 }\end{array}$} & Catastrófico & 1 & 1 & 2 & 4 & 7 & 11 \\
\hline & Mortalidad & 2 & 3 & 5 & 8 & 12 & 16 \\
\hline & Permanente & 3 & 6 & 9 & 13 & 17 & 20 \\
\hline & Temporal & 4 & 10 & 14 & 18 & 21 & 23 \\
\hline & Menor & 5 & 15 & 19 & 22 & 24 & 25 \\
\hline & & & A & B & C & D & E \\
\hline & & & Común & Ha sucedido & Podría suceder & Raro que suceda & Prácticamente imposible que suceda \\
\hline & & & & & FRECUENCIA & & \\
\hline
\end{tabular}




\section{RESULTADOS}

\subsection{Análisis e interpretación de resultados}

El estudio de la muestra seleccionada se realizó en la empresa Stierlift por ser una de la más importantes del país, es líder en el transporte de carga pesada y sobredimensionada y trabajos de maniobra convencional.

La empresa de muestra tiene 20 trabajadores en total que participan en los trabajos en los diferentes trabajos de maniobra (Ver Tabla 3).

De acuerdo con la metodología descrita, se tomaron en cuenta 3 trabajos de maniobra convencional más representativos de la empresa Stierlift SA, en donde se combinan diferentes elementos de maniobra y formas de ejecución. Para lo cual cada trabajo se dividió en actividades y posteriormente en tareas. Se identificó los peligros y riesgos de cada una de las tareas de los 3 trabajos de maniobra convencional seleccionados.

Habiendo determinado los peligros y riesgos de cada trabajo de maniobra convencional, luego se obtuvo el resultado de la información de antecedentes de accidentes y enfermedades ocupacionales en los trabajos de maniobra convencional de la empresa Stierlift SA, por medio de la recopilación de datos brindados por personal de la empresa y también por medio de la entrevista con el medico ocupacional y responsable del área de QHSE; esto con la finalidad de determinar las consecuencias y realizar la valoración de los riesgos

Se determinó las consecuencias de cada riesgo basado en la recopilación de información y a las entrevistas con los involucrados en el proceso; para luego realizar la valoración de los riesgos según la metodología descrita.

Finalmente, se determina su significancia, según la metodología utilizada los valores de nivel de riesgo que se encuentren entre 1 a 8 son considerados riesgo alto o significativo y se representan de color rojo; de esta forma en cada uno de los 3 trabajos de maniobra convencional seleccionados.

Se determinaron los riesgos significativos, según la evaluación de riesgos para cada uno de los trabajos.

En base a los resultados obtenidos, como riesgos significativos en los tres trabajos de maniobra convencional, se elaboró una gráfica comparativa, que muestra la cantidad de riesgos significativos en cada trabajo y cuál de los tres presenta la mayor cantidad (Ver Figura 1).

También se determinó un resumen de los valores en cantidad de los tipos de riesgos; alto, medio y bajo; encontrados en cada uno de los 3 trabajos de maniobra convencional seleccionados para el presente estudio. (Ver Figura 2, Figura 3 y Figura 4)

\subsection{Demostración de la hipótesis}

Una evaluación de los riesgos laborales si influye significativamente ya que con una evaluación de riesgos para los trabajos de maniobra convencional podemos determinar la magnitud de los riesgos ya que según la metodología aplicada basada en el DS024-2016 EM, se les da una ponderación con valor numérico y se clasifica según rangos para determinar su significancia o criticidad.

Al darle un valor numérico a los riesgos podremos determinar cual tiene mayor o menor magnitud y luego clasificarlos, los que se encuentran en un nivel alto son considerados riesgos de mayor criticidad o significativos.

Al realizar una evaluación de riesgos se determina las consecuencias generados por los riesgos identificados a los que se exponen los trabajadores, estas consecuencias son basadas en la metodología aplicada usando la observación, la entrevista, los antecedes de accidentes y enfermedades ocupacionales y finalmente un criterio de evaluador, esto se observa en los resultados.

Tabla 2. Niveles de Riesgo

\begin{tabular}{|l|l|l|c|}
\hline \multicolumn{2}{|c|}{ Nivel de Riesgo } & \multicolumn{1}{|c|}{ Descripción } & Plazo de Medida Correctiva \\
\hline & ALTO & $\begin{array}{l}\text { Riesgo intolerable, requiere controles inmediatos. Si no se puede controlar el PELIGRO se paralizan } \\
\text { los trabajos operacionales en la labor. }\end{array}$ & $0-24$ HORAS \\
\hline MEDIO & $\begin{array}{l}\text { Iniciar medidas para eliminar/reducir el riesgo. Evaluar si la acción se puede ejecutar de manera } \\
\text { inmediata }\end{array}$ & $0-72$ HORAS \\
\hline BAJO & Este riesgo puede ser tolerable. & 1 MES \\
\hline
\end{tabular}

Fuente. Datos tomados del DS024-2016 EM

Tabla 3. Distribución \% de la fuerza laboral de maniobra

\begin{tabular}{|c|c|c|c|c|c|c|}
\hline \multirow[b]{2}{*}{ Personal } & \multirow[b]{2}{*}{ \# de trabajadores } & \multirow[b]{2}{*}{$\%$} & \multicolumn{2}{|c|}{ Distribución } & \multirow[b]{2}{*}{ Horas de trabajo } & \multirow[b]{2}{*}{ Turno } \\
\hline & & & $20-40$ & $>40$ & & \\
\hline Supervisor de Maniobra & 2 & 10 & 0 & 2 & 12 horas & Rotativo \\
\hline Maniobrista & 1 & 5 & 0 & 1 & 12 horas & Rotativo \\
\hline Operador & 7 & 35 & 5 & 2 & 12 horas & Rotativo \\
\hline Ayudante de maniobra & 10 & 50 & 8 & 2 & 12 horas & Rotativo \\
\hline Total & 20 & 100 & 13 & 7 & & \\
\hline
\end{tabular}




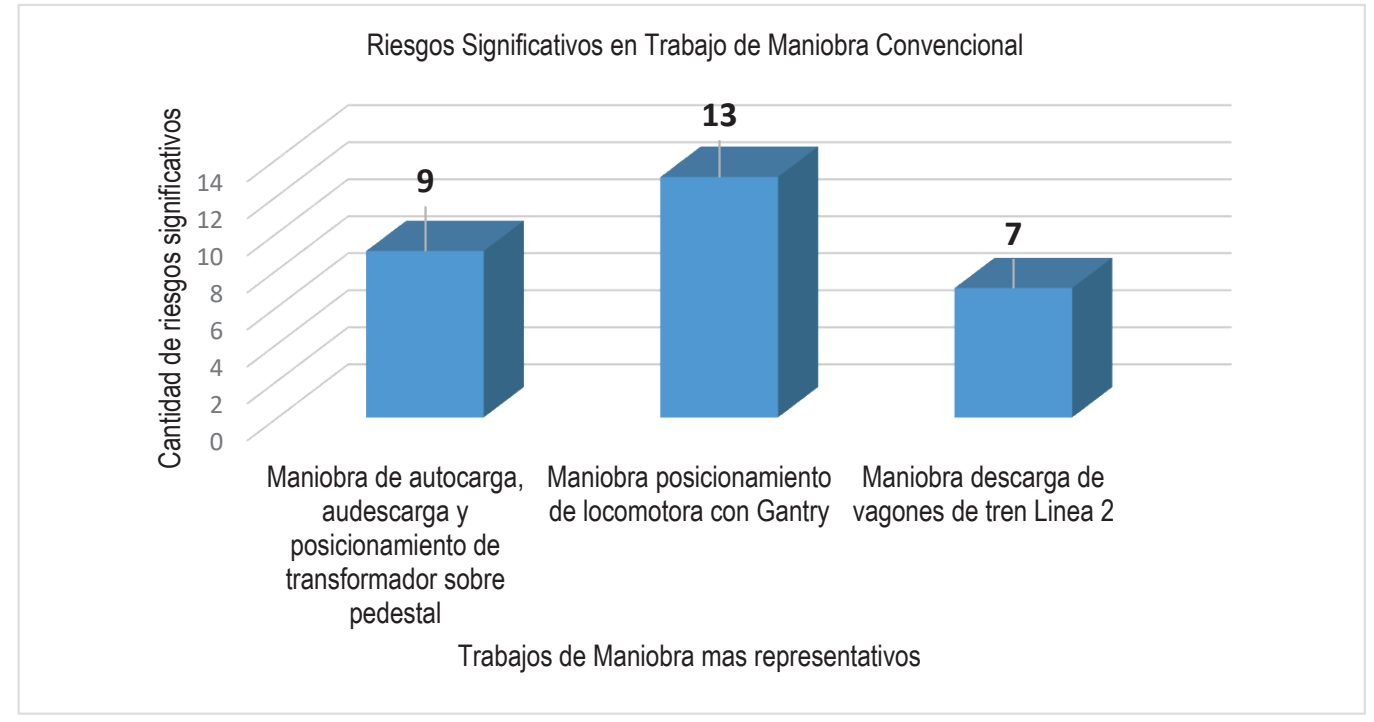

Figura 1. Riesgos Significativos en Trabajo de Maniobra.

\section{Resumen Maniobra de autodescarga y posicionamiento de transformador sobre pedestal}

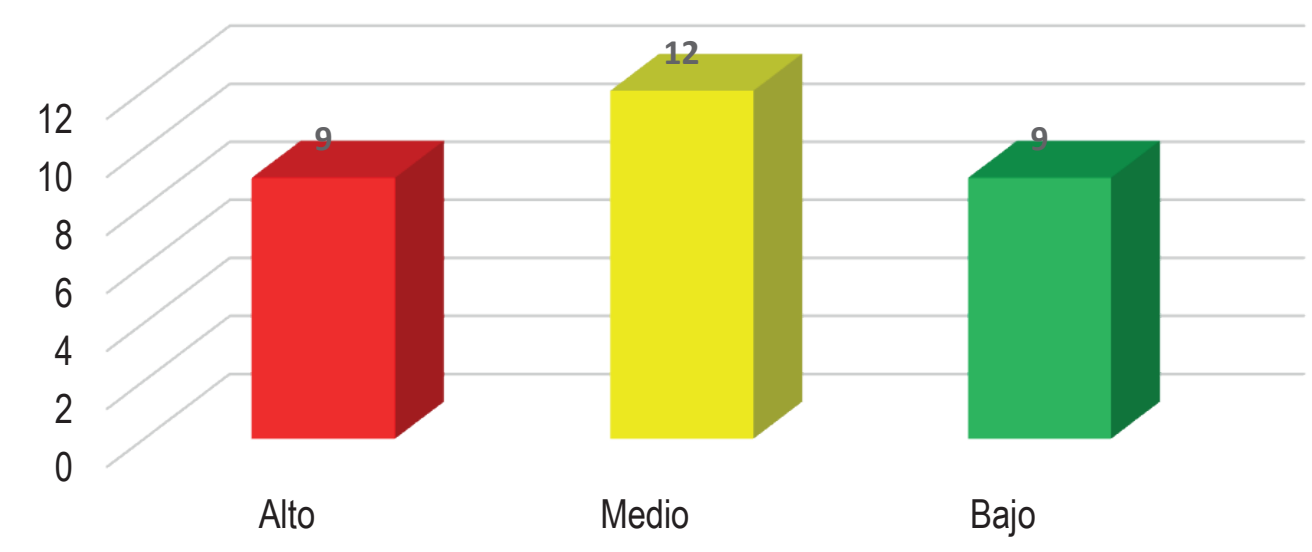

Figura 2. Resumen Maniobra de autodescarga y posicionamiento de transformador sobre pedestal.

\section{Resumen Maniobra posicionamiento de locomotora con Gantry}

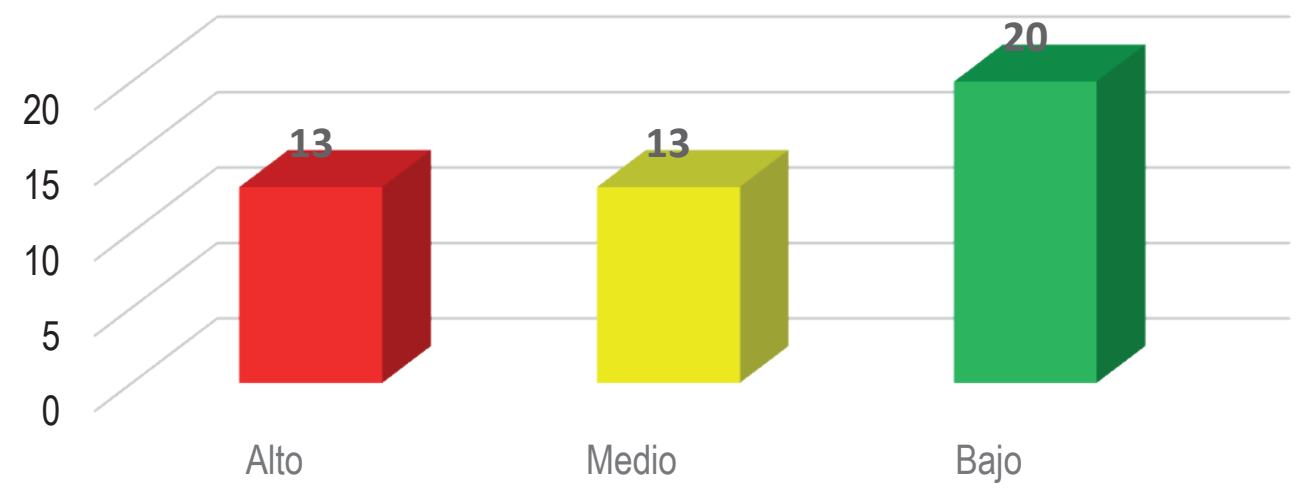

Figura 3. Resumen Maniobra posicionamiento de locomotora con Gantry. 


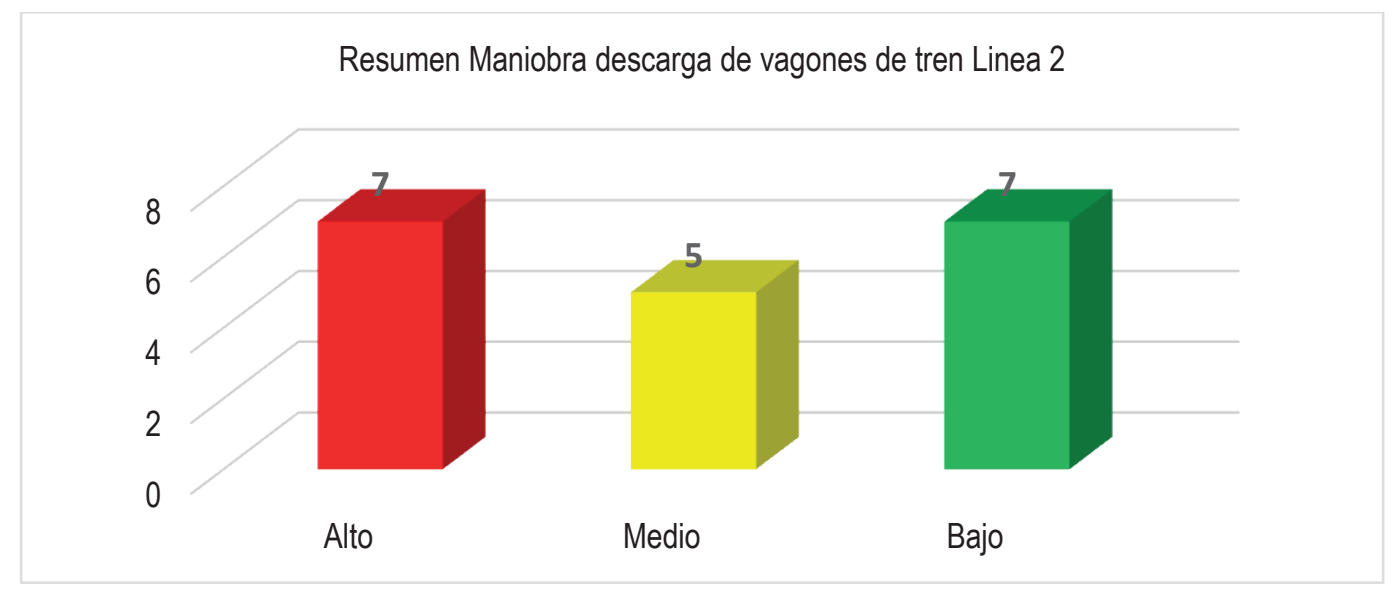

Figura 4. Resumen Maniobra descarga de vagones de tren Línea 2.

Habiendo determinado los riesgos y las consecuencias se procede a la valoración según la metodología DS0242016 EM, en base a los resultados obtenidos se podrá proponer un plan de acción de acuerdo con las medidas de control y según la jerarquía de controles, orientado a la mitigación de los riesgos y si se cumplen los controles que se establecen, se realizará un trabajo más seguro que reducirá la tasa de accidentes.

\section{DISCUSIÓN}

Al hacer la comparación de las evaluaciones de los riesgos significativos en los tres trabajos de maniobra se obtuvo como resultado que la maniobra de Gantry presenta el mayor número de riesgos significativos con un total de 13 y la maniobra de descarga de vagones es la que menor riesgos significativos presenta con un total de 7 , estos resultados nos ayudan a determinar el trabajo de maniobra más crítico y así plantear controles específicos. También si lo comparamos con el estudio monográfico elaborado por la Consejería de Economía y empleo de España (2008) sobre los riesgos en los trabajos de maniobra, se corrobora similitud en los resultados finales donde este autor determina que los riesgos que se generan son de carácter critico en su mayoría por lo que propone controles y formas de trabajo para evitar accidentes.

\section{CONCLUSIONES}

Se concluye que por medio de la evaluación de riesgos que se realizó a las actividades de maniobra convencional, que las tareas que desempeñan los trabajadores representan de un nivel de riesgo de medio hasta alto ya que en un promedio de los tres trabajos de maniobra considerados en el presente estudio se obtiene un $32 \%$ de riesgos de nivel alto, por lo tanto, los puestos de trabajo involucrados presentan una mayor exposición a accidentes.

\section{REFERENCIAS}

Consejería de Economía y empleo de España. (2008). Maniobras de carga y descarga en el ámbito del transporte de mercancias por carretera. http://
trabajoyprevencion.jcyl.es/web/jcyl/TrabajoYPrevencion/ es/Plantilla100Detalle/1284173133969/_/1284173420155/ Redaccion

Del Águila Guerrero, E. F. (2020). Influencia de la aplicación de la norma G050 en el clima de seguridad de las obras de construcción del distrito de Surquillo. Revista Del Instituto de Investigación de La Facultad de Ingeniería Geológica, Minera, Metalúrgica y Geográfica, 23(45), 3-10. https:// doi.org/10.15381/iigeo.v23i45.18044

Diario El Peruano. (2016). Decreto Supremo Nº 024-2016-EM. Ministerio de Energía y Minas. https://busquedas.elperuano. pe/normaslegales/aprueban-reglamento-de-seguridad-ysalud-ocupacional-en-mine-decreto-supremo-n-024-2016em-1409579-1/

Falconi Agapito, F., \& Romero Baylon, A. (2020). Las micro, pequeña y mediana empresa y sus adaptación a la normativa de seguridad y la salud en el trabajo. Revista Del Instituto de Investigación de La Facultad de Ingeniería Geológica, Minera, Metalúrgica y Geográfica, 23(45), 17-28. https:// doi.org/10.15381/iigeo.v23i45.18058

Flores Arévalo, P. R. (2019). Enfoque integrado de la administración en Salud, Seguridad Ocupacional y Ambiente en los Laboratorios Clínicos Integrated Management System in Safety, Occupational Health and Environment for Clinical Laboratories. Revista Del Instituto de Investigación FIGMMG-UNMSM, 22(43), 22, 81-92. https://revistasinvestigacion.unmsm.edu.pe/index.php/ iigeo/article/view/16692/14255

Hernández Sampieri, R., Fernández Collado, C., María del Pilar Baptista Lucio, D., \& Méndez Valencia Christian Paulina Mendoza Torres, S. (2014). Metodología de la investigación (6ta Ed.). https://www.esup.edu.pe/descargas/ perfeccionamiento/PLAN LECTOR PROGRAMA ALTO MANDO NAVAL 2020/2. Hernandez, Fernandez y BaptistaMetodología Investigacion Cientifica 6ta ed.pdf

Stierlift SA. (2019). Transporte especializado, grúas. Maniobras. http://www.stierlift.pe/servicios/ingenieria/maniobrasespeciales/

The Bechtel Equipment Operations Department Rigging. (2002). Bechtel Rigging Handbook (Second Edition). 190. https:// docplayer.net/18190633-Bechtel-rigging-handbooksecond-edition.html 